\title{
Epidural abscess and subdural empyema
}

\author{
JOHN A. SELLICK, Jr, DO
}

\begin{abstract}
Epidural abscess and subdural empyema are serious intracranial infections that result in significant morbidity and mortality. Frequently, they are secondary to sinusitis or middle ear disease, and the bacteria involved are inhabitants of the upper respiratory tract. Symptoms may be mild and mimic the symptoms of the underlying infection. However, especially with subdural empyema, alteration in the level of consciousness and focal neurologic deficits are common. Morbidity and mortality are minimized by early diagnosis, which is best made with computed tomography scanning, and proper therapy, which consists of surgical drainage and administration of appropriate antimicrobials. It is important that primary care physicians be aware of the clinical features of these potentially fatal complications of common infections.
\end{abstract}

"Intracranial suppurations" is an imprecise term that refers to pyogenic infections occurring in or around the brain and its lining structures. Two such processes are cranial epidural abscess and subdural empyema. The clinical features, treatment, and outcome of each is different. Although these secondary infections are uncommon, they occur as complications of common upper respiratory tract infections. Primary care physicians therefore should be aware of their clinical features.

\section{Epidural abscess}

\section{Case 1}

A previously healthy 13-year-old boy began to experience cough, sneezing, and rhinorrhea 2 weeks prior to admission. He was seen by his family physician, who made a clinical diagnosis of sinusitis and prescribed amoxicillin/clavulanate potassium, which the patient took for the week before admission. The symptoms continued, however, and frontal headache developed. On the day prior to admission, he became febrile and lethargic. $\mathrm{He}$ had a generalized tonic-clonic seizure and was brought to the Children's Hospital of Buffalo.

At the time of physical examination, he was afebrile but somnolent. There was mild periorbital edema. The findings of the remainder of the examination were within normal limits. Laboratory studies revealed a WBC count of $17,400 / \mathrm{cu} \mathrm{mm}$, with $68 \%$ polymorphonuclear cells, $16 \%$ band neutrophils, $10 \%$ lymphocytes, $5 \%$ monocytes, and $1 \%$ eosinophils. The sedimentation rate was $77 \mathrm{~mm} / \mathrm{h}$, and urinalysis results and chemistry profile were within normal limits. A computed tomography (CT) scan of the head revealed opacified frontal sinuses and a small frontal epidural abscess.

The patient underwent drainage of the frontal sinus, which yielded Streptococcus morbillorum and Peptostreptococcus micros on anaerobic culture. Aerobic and fungal cultures were negative. The patient was started on a regimen of chloramphenicol and showed rapid clinical improvement. Repeat CT scan showed no increase in the size of the abscess. The patient was discharged on chloramphenicol therapy. At 6 month follow-up, he was still well.

\section{Discussion}

Epidural abscess is a recognized complication of acute or chronic frontal sinusitis ${ }^{1-3}$ or middle ear disease. ${ }^{4-6}$ Since epidural abscess is uncommon, it is difficult to determine the actual incidence from the available literature. In two series from university centers that contained sufficient cases to comment on, there was about one case each 3 to 5 years. ${ }^{5,7}$ In contrast, in a medically impoverished population in South Africa, there were 49 cases of epidural abscess discovered among patients attending an ear, nose, and throat outpatient clinic between 1978 and $1983 .^{6}$

The pathogenesis of epidural abscess involves the spread of infection from the sinus or mastoid to the epidural space by way of valveless diploic veins, or less commonly, erosion of the posterior wall of the frontal sinus or the mastoid bone with resultant contiguous spread. ${ }^{1,4,8}$ The infection may spread from the epidural space to the subdural space or the brain parenchyma. This spread may be as a result of destruction of the dura, but the intertwined venous system of these contiguous structures is probably the more likely route. ${ }^{1}$

The bacteria that are commonly involved in epidural abscess are listed in Table 1 in approximate order of occurrence. Aerobic streptococci and anaerobic gram-positive cocci and gram-negative bacilli predominate, with a small number of episodes due to staphylococci and Haemophilus species. An- 


\begin{tabular}{|c|c|}
\hline \multicolumn{2}{|c|}{$\begin{array}{l}\text { Table } 1 \\
\text { Microbiologic Profile in Subdural Empyema } \\
\text { and Epidural Abscess* }\end{array}$} \\
\hline Bacterial involvement & $\begin{array}{c}\text { Percentage } \\
\text { of cases }\end{array}$ \\
\hline $\begin{array}{l}\text { Aerobic streptococci } \\
\text { Alpha-hemolytic } \\
\text { Beta-hemolytic } \\
\text { Pneumococci }\end{array}$ & $30-50$ \\
\hline $\begin{array}{l}\text { Anaerobes } \\
\text { Peptococcus species } \\
\text { Peptostreptococcus species } \\
\text { Bacteroides species } \\
\text { Fusobacterium species }\end{array}$ & $20-40$ \\
\hline $\begin{array}{l}\text { Staphylococci } \\
\text { S aureus } \\
\text { S epidermidis }\end{array}$ & $15-25$ \\
\hline Haemophilus & 5 \\
\hline Aerobic/facultative gram-negative rods & 5 \\
\hline
\end{tabular}

aerobes may be less common in older studies because of inadequate anaerobic culture techniques. 9,10

The clinical presentation of epidural abscess is difficult to distinguish from that of the underlying infection. Patients may complain of fever, headache, upper respiratory tract symptoms, or local tenderness over the sinuses or mastoid. Focal neurologic deficits generally do not occur unless the abscess is large. $1,6,7$

Presurgical diagnosis was possible only with arteriography in the past. ${ }^{11}$ Computed tomography scanning appears to have supplanted arteriography, and it should be possible to localize the abscess prior to surgery in most cases. ${ }^{12-14}$ Epidural abscess is described as showing an extradural mass with a thick, dense ring of enhancement when contrast is administered. ${ }^{12-14}$ However, the ring may not be distinct early in the course of infection, and it may persist for up to 2 weeks postoperatively. ${ }^{13}$ There is concern over reports of false-negative scans $^{7-8}$; however, newer generation scanners may alleviate this problem. ${ }^{8}$

Treatment usually consists of antimicrobial therapy and surgical drainage of the abscess and the affected sinus or mastoid. ${ }^{1,7}$ The patient in case 1 responded to a less aggressive approach, probably because the epidural abscess was small and centrally located, and his level of consciousness was not significantly depressed. Appropriate antibiotic therapy should be administered early and continued for a prolonged period of time, particularly if there is associated osteomyelitis or residual fluid collection.

On the basis of the bacteriologic picture described heretofore, appropriate empiric therapy should include coverage of aerobic and anaerobic upper airway flora. Penicillin may be adequate in this regard. However, concern about penicillin resistance among some upper airway anaerobes has been expressed. ${ }^{9}$ In this latter situation it would be reasonable to use metronidazole or chloramphenicol in addition to penicillin. In children, Haemophilus coverage should be included, which can be accomplished with ampicillin, chloramphenicol, cefuroxime, or a third-generation cephalosporin. It also may be necessary to add antistaphylococcal coverage. Empiric therapy may be modified on the basis of results of Gram's staining of specimens obtained at the time of surgery. Ultimately, therapy should be based on the results of properly obtained aerobic and anaerobic cultures of abscess material. ${ }^{8}$ The prognosis of this disease is good, with low morbidity and mortality when managed appropriately. 6,7

This case of epidural abscess is typical of many cases described in the literature. The patient had a clinical picture that suggested sinusitis without any evidence of neurologic impairment, but appropriate therapy did not resolve his symptoms. The subsequent development of headache and seizure led to the diagnostic CT scan. The patient underwent drainage of the frontal sinuses and received a prolonged course of antibiotics, resulting in cure. In an analogous case, nonsurgical treatment of a small, intrahemispheric subdural empyema has been reported. ${ }^{15}$ This should not be construed as a "trend," but there may be a subset of neurologically intact patients with small epidural collections of pus who may be cured with medical therapy alone.

\section{Subdural empyema}

\section{Case 2}

A 45-year-old man with a history of chronic alcoholism was brought to the Emergency Department at the Buffalo Veterans Administration Medical Center after suffering a partial, complex, left-sided seizure that progressed to a generalized tonic-clonic-tonic seizure. A small, chronic, right parietal, subdural hematoma had been diagnosed 6 months before admission. His medical history was also positive for alcoholic liver disease, seizure disorder, hypertension, and Wernicke's encephalopathy. 
On admission, he was unarousable and restless. Vital signs were normal and he was afebrile. Left-sided facial palsy was evident. There were no spontaneous or purposeful movements on the left side of his body; toes were upgoing bilaterally, and no left-sided deep tendon reflexes could be elicited. Routine admitting laboratory studies yielded essentially normal results except for a WBC count of $18,400 / \mathrm{cu}$ mm with a normal differential count.

The patient was taken to the operating room on the night of admission for anticipated drainage of a subdural hematoma. Right occipital bur holes were placed with subsequent drainage of $200 \mathrm{~mL}$ of thick, purulent material from the epidural and subdural spaces. This material yielded growth of Streptococcus pneumoniae. Chloramphenicol (500 $\mathrm{mg}$ IV q6h) and penicillin (4 million units $\mathrm{q} 4 \mathrm{~h}$ ) treatment was started and the latter regimen was continued once culture results were known.

Later in the hospitalization, a CT scan of the mastoids showed opacification of the right mastoid air cells, sclerosis of the tip of the right mastoid, and an old fracture of the right occipital bone. Tympanometry showed decreased motion of the right tympanic membrane, and the patient later underwent insertion of a tympanostomy tube on the right with drainage of sterile fluid.

The patient recovered from surgery, and he remained without sequelae when seen at 18-month follow-up. He received a total of 6 weeks of parenteral penicillin treatment.

\section{Case 3}

A 59-year-old woman was referred by her family physician at the Buffalo General Hospital with a 7- to 10-day history of watery diarrhea and progressive generalized weakness. She denied any history of headache, focal neurologic deficit, or sinus disease. The remainder of her past history was noncontributory.

At the time of admission, the patient was afebrile and vital signs were normal. Except for bilateral periorbital edema, the findings of physical examination were within normal limits. Laboratory studies on admission revealed a WBC count of $19,800 / \mathrm{cu} \mathrm{mm}$ with $61 \%$ polymorphonuclear neutrophil leukocytes, and $22 \%$ band neutrophils. Serum values were as follows: sodium, $125 \mathrm{mEq} / \mathrm{L}$; potassium, $3.0 \mathrm{mEq} / \mathrm{L}$; chloride, $91 \mathrm{mEq} / \mathrm{L}$; and bicarbonate, $31 \mathrm{mEq} / \mathrm{L}$.

The patient was given intravenous fluids, and a workup for the diarrhea was started. Soon after admission, headache and fever developed. A spinal tap revealed a WBC count of $1000 / \mathrm{cu} \mathrm{mm}, 78 \%$ of which were neutrophils. The CSF glucose level was $53 \mathrm{mg} / \mathrm{dL}$. Results of culture and bacterial antigen studies were negative. A CT scan of the head showed opacification of the frontal sinuses and a right frontal subdural fluid collection. She was started on a regimen of cefuroxime (1.5 g IV q6h) and metronidazole ( $500 \mathrm{mg}$ IV q6h).

The patient underwent placement of right frontal bur holes with drainage of thick, yellow material. This was sterile on aerobic culture but grew $P$ micros and $E u$ - bacterium species. Simultaneous blood cultures grew $P$ micros, Eubacterium species, and an unidentified, anaerobic gram-negative rod. The patient never regained consciousness postoperatively and neurologic function progressively declined. She died on the 16th hospital day.

\section{Discussion}

Subdural empyema is more common than epidural abscess and, as a result, the body of clinical literature concerning this infection is larger., $5,7,9,16-23 \mathrm{Ta}$ ble 2 shows the incidence of subdural empyema that has been reported in several series. Each of these studies was done at a large university center where, in most instance, approximately one case per year was seen during the indicated time periods. Two reports from a regional neurosurgery center in Great Britain, overlapping each other by several years, recount two to three cases per year. ${ }^{16,20}$

In the series of the past 20 years, most cases are described as being secondary to frontal sinusitis, ${ }^{7,16,18-21}$ though many patients do not give a history of chronic sinusitis symptoms. ${ }^{19,21}$ A significant number of cases still are related to otogenic foci. $4,5,10,16,20,22$ However, the latter are probably less common in the antibiotic era since appropriate therapy has greatly reduced the progression of middle ear disease to mastoiditis. ${ }^{24}$ Subdural empyema also is recognized as a postoperative complication of cranial neurosurgical procedures, ${ }^{5,7,10,17,19}$ and occasionally is seen after trauma. ${ }^{15,17}$ Hematogenous seeding of the subdural space ${ }^{5,7,19,20}$ and a preexisting subdural hematoma ${ }^{17}$ have been suggested in several studies. However, it is difficult to know whether sinusitis, otitis, and mastoiditis were adequately ruled out.

The pathogenesis of subdural empyema is analogous to that of epidural abscess. An existing epidural abscess may erode through the dura to infect the subdural space, though the most likely route of spread appears to be by way of the valveless venous network as previously described. ${ }^{1}$ Once organisms are introduced into the subdural space, the infection expands rapidly and may spread along the convexity of the cerebral hemisphere to the parafalcial areas and beneath the falx to the opposite cortical surface. ${ }^{1,16,18,19}$ The purulent material may also spread to the posterior fossa. ${ }^{22}$

The bacteriologic profile of subdural empyema is outlined in Table 1. Aerobic streptococci and anaerobes account for $50 \%$ to $75 \%$ of infections in recent series. Infections due to Staphylococcus aureus and coagulase-n'egative staphylococci may be more common in patients with subdural empyema than those with epidural abscess. ${ }^{10,16,19}$ It is difficult to determine the significance of this latter ob- 
Table 2

Incidence of Subdural Empyema (SDE) at Large University Centers

\begin{tabular}{|c|c|c|c|}
\hline Author and reference & $\begin{array}{l}\text { Years } \\
\text { included }\end{array}$ & $\begin{array}{l}\text { No. of } \\
\text { cases }\end{array}$ & Comments \\
\hline Kiser and Kendig $(1963)^{5}$ & $1942-1961$ & 11 & Includes 5 cases with both SDE and epidural abscess \\
\hline Smith and Hendrick $(1983)^{7}$ & $1954-1981$ & 22 & Includes 3 cases with both SDE and epidural abscess \\
\hline Bhandari and Sarkari $(1970)^{16}$ & $1954-1968$ & 37 & Regional neurosurgical center \\
\hline Bannister et al $(1981)^{20}$ & $1954-1979$ & 66 & $\begin{array}{l}\text { Regional neurosurgical center (overlaps cases } \\
\text { reported by Bhandari and Sarkari }{ }^{16} \text { ) }\end{array}$ \\
\hline Kaufman et al $(1975)^{19}$ & $1967-1974$ & 17 & All underlying diseases \\
\hline Kaufman et al $(1983)^{21}$ & $1967-1982$ & 17 & Sinusitis-related cases \\
\hline Ariza et al (1986) ${ }^{10}$ & $1975-1984$ & 9 & Includes 5 cases with both SDE and epidural abscess \\
\hline Brook et al $(1980)^{9}$ & $1977-1980$ & 4 & Sinusitis-related cases \\
\hline
\end{tabular}

servation since all of the cited studies are retrospective and the number of reports of epidural abscess is small. Ariza and coauthors ${ }^{10}$ documented associated bacteremia in four of their patients, which they suggested was due to septic thrombophlebitis of the lateral sinus. In all four instances, bacteremia was due to multiple anaerobic organisms that were also grown from the purulent intracranial material.

Early clinical features of subdural empyema may be similar to those of sinusitis, mastoiditis, or epidural abscess. However, a high incidence of significant neurologic findings, such as papilledema, hemiparesis, seizures, and alterations in consciousness, has been reported. ${ }^{16,18-20}$ These findings are secondary to cerebral cortical irritation or increased intracranial pressure. ${ }^{8}$ Since the subdural space does not limit the spread of infection, cortical compression and increased intracranial pressure develop rapidly. Death ensues from transtentorial herniation of the brain or uncontrolled infection. ${ }^{19}$

In subdural empyema as in epidural abscess, CT scanning has replaced cerebral angiography as the most important diagnostic tool. Subdural empyema typically is visualized as a hypodense crescentic or elliptic area along the skull or the falx.$^{12,23}$ Weisberg ${ }^{23}$ suggests that the reasons for possible failure of CT to delineate subdural empyema in some prior reports ${ }^{20,21,25}$ are as follows: (1) use of older scanners with low resolution; (2) slow scan times producing motion artifact; (3) scan produced without contrast; (4) interpretative errors; (5) no coronal scans included; and (6) performance of scan before development of empyema.
With the use of newer generation scanners and by using contrast enhancement, CT should be highly accurate, except possibly when done early in the course of infection, prior to the development of a fluid collection. ${ }^{23}$ Lumbar puncture is hazardous in this situation because of the increased intracranial pressure ${ }^{19}$ but, if it is performed, the CSF has an elevated WBC count and protein level, normal glucose level, and negative Gram's stain and culture.$^{5,19}$ One report of nine cases indicated that electroencephalography occasionally may be more sensitive than CT. ${ }^{26}$ Abnormalities reported in essentially all cases were focal zeta waves, unilateral depression of cortical activity, and diffuse slowing of background activity. Other reports have not shown electroencephalography to be reliable. ${ }^{19}$

Subdural empyema has been considered a true "neurosurgical emergency," since without surgical intervention the disease has a high mortality. ${ }^{5,7,16-22}$ In a collective review, Bannister and coworkers ${ }^{20}$ compared craniotomy versus bur holes for the drainage of subdural empyema. They strongly suggested that open surgical drainage had a significant benefit; however, the data were collected in an uncontrolled manner and there were many potential areas for bias. Therefore, no definite recommendations can be made in regard to the best form of surgical therapy. Likewise, the role of indwelling drains and antibiotic irrigation is not defined ${ }^{8}$

Successful nonsurgical therapy for subdural empyema has been reported, ${ }^{15,27}$ including several patients who had large collections or depressed levels of consciousness. ${ }^{27}$ Essential to this approach 
is close clinical follow-up of the patients, serial CT scans of the head, and high-dose parenteral antibiotics. ${ }^{27}$ The desire to avoid surgery should be tempered with the weight of experience and understanding of the aggressive nature of this infection.

Antimicrobial therapy should be started as soon as the diagnosis is made. Empiric therapy should be directed toward the aerobic streptococci and anaerobes, with the same therapy that was recommended before. Antistaphylococcal coverage should also be added until Gram's staining and culture results are obtained. In children, coverage for Haemophilus should be included. The mortality rate for subdural empyema with early and adequate therapy is approximately $20 \% .^{19-21}$ Unfortunately, some survivors may be left with permanent neurologic sequelae, primarily seizures, hemiparesis, and dysphasia. ${ }^{19,28}$ Patients who have a depressed level of consciousness when first seen have mortality rates that are two to three times higher than patients who are responsive. ${ }^{20}$

These two cases illustrate important points concerning subdural empyema. Case 2 is an instance in which we lacked information regarding prodromal illness, other than a history of subdural hematoma. The patient's obvious focal neurologic findings and positive CT scan dictated the necessity for surgical intervention. Combined epidural abscess and subdural empyema were found at surgery, and drainage was accomplished successfully. Although some reports suggest hematogenous seeding of an existing chronic subdural hematoma as the cause, this patient had documented, untreated middle ear and mastoid disease, which was the likely source of infection.

In Case 3 , the patient had nonspecific prodromal symptoms and no past history of sinusitis. Unfortunately, the unexplained periorbital edema did not prompt CT scanning. The diagnosis was not made until the patient had undergone significant change in mental status. It is not known whether the lumbar puncture contributed to her death. She had polymicrobial anaerobic bacteremia as well.

1. Remmler D, Boles R: Intracranial complications of frontal sinusitis. Laryngoscope 1980;90(pt 1):1814-1824.

2. Bluestone CD, Steiner RE: Intracranial complications of acute frontal sinusitis. South Med J 1965;58:1-10.

3. Sable NS, Hengerer A, Powell KR: Acute frontal sinusitis with intracranial complications. Pediatr Infect Dis 1984;3:58-61.

4. Gower D, McGuirt WF: Intracranial complications of acute and chronic infectious ear disease: A problem still with us. Laryngoscope 1983;93:1028. 1033.

5. Kiser JL, Kendig JH: Intracranial suppuration: A review of 139 consecutive cases with electron-microscopic observations on three. $J \mathrm{Neu}$ rosurg 1963;20:494-511.

6. Samuel J, Fernandes CMC, Steinberg JL: Intracranial otogenic complications: A persisting problem. Laryngoscope 1986;96:272-278.

7. Smith HP, Hendrick EB: Subdural empyema and epidural abscess in children. $J$ Neurosurg 1983;58:392-397.

8. Silverberg AL, DiNubile MJ: Subdural empyema and cranial epidural abscess. Med Clin North Am 1985;69:361-374.

9. Brook I, Friedman EM, Rodriguez WJ, et al: Complications of sinusitis in children. Pediatrics 1980;66:568-572.

10. Ariza J, Casanova A, Viladrich PF, et al: Etiological agent and primary source of infection in 42 cases of focal intracranial suppuration. $J$ Clin Microbiol 1986;24:899-902.

11. Handel SF, Klein WC, Kim YW: Intracranial epidural abscess. $R a$ diology 1974;111:117-120.

12. Kaufman DM, Leeds NE: Computed tomography (CT) in the diagnosis of intracranial abscesses: Brain abscess, subdural empyema, and epidural empyema. Neurology 1977;27:1069-1073.

13. Blaquière RM: The computed tomographic appearances of intra- and extracerebral abscesses. Br J Radiol 1983;56:171-181.

14. Moseley IF, Kendall BE: Radiology of intracranial empyemas, with special reference to computed tomography. Neuroradiology 1984;26:333345 .

15. Mauser HW, Ravijst RAP, Elderson A, et al: Nonsurgical treatment of subdural empyema: Case report. J Neurosurg 1985;63:128-130.

16. Bhandari YS, Sarkari NBS: Subdural empyema: A review of 37 cases. J Neurosurg 1970;32:35-39.

17. Coonrod JD, Dans PE: Subdural empyema. Am J Med 1972;53:8591

18. Farmer TW, Wise GR: Subdural empyema in infants, children and adults. Neurology 1973;23:254-261.

19. Kaufman DM, Miller MH, Steigbigel NH: Subdural empyema: Analysis of 17 recent cases and review of the literature. Medicine 1975;54:485498.

20. Bannister G, Williams B, Smith S: Treatment of subdural empyema. $J$ Neurosurg 1981;55:82-88.

21. Kaufman DM, Litman N, Miller MH: Sinusitis: Induced subdural empyema. Neurology 1983;33:123-132.

22. Morgan DW, Williams B: Posterior fossa subdural empyema. Brain 1985;108:983-992.

23. Weisberg L: Subdural empyema: Clinical and computed tomographic correlations. Arch Neurol 1986;43:497-500.

24. Klein JO: Otitis externa, otitis media, mastoiditis, in Mandell GL, Douglas RG Jr, Bennett JE (eds): Principles and Practice of Infectious Disease, ed. 2. New York, John Wiley \& Sons, 1985, pp 364-369.

25. Dunker RO, Khakoo RA: Failure of computed tomographic scanning to demonstrate subdural empyema. JAMA 1981;246:1116-1118.

26. Mauser HW, Van Heffelen AC, Tulleken CAF: The EEG in the diagnosis of subdural empyema. Electroenceph Clin Neurophysiol 1986;64:511516.

27. Leys D, Destee A, Petit H, et al: Management of subdural intracranial empyemas should not always require surgery. Neurol Neurosurg Psychiatry 1986;49:635-639.

28. Cowie R, Williams B: Late seizures and morbidity after subdural empyema. J Neurosurg 1983;58:569-573.

From the department of medicine, division of infectious disease, University of Buffalo School of Medicine and Biomedical Sciences, SUNY, Buffalo, NY.

Reprint requests to Dr Sellick, department of medicine, Buffalo General Hospital, 100 High St, Buffalo, NY 14201. 


\section{THE NEWLY \\ DIAGNOSED HYPERTENSIVE}

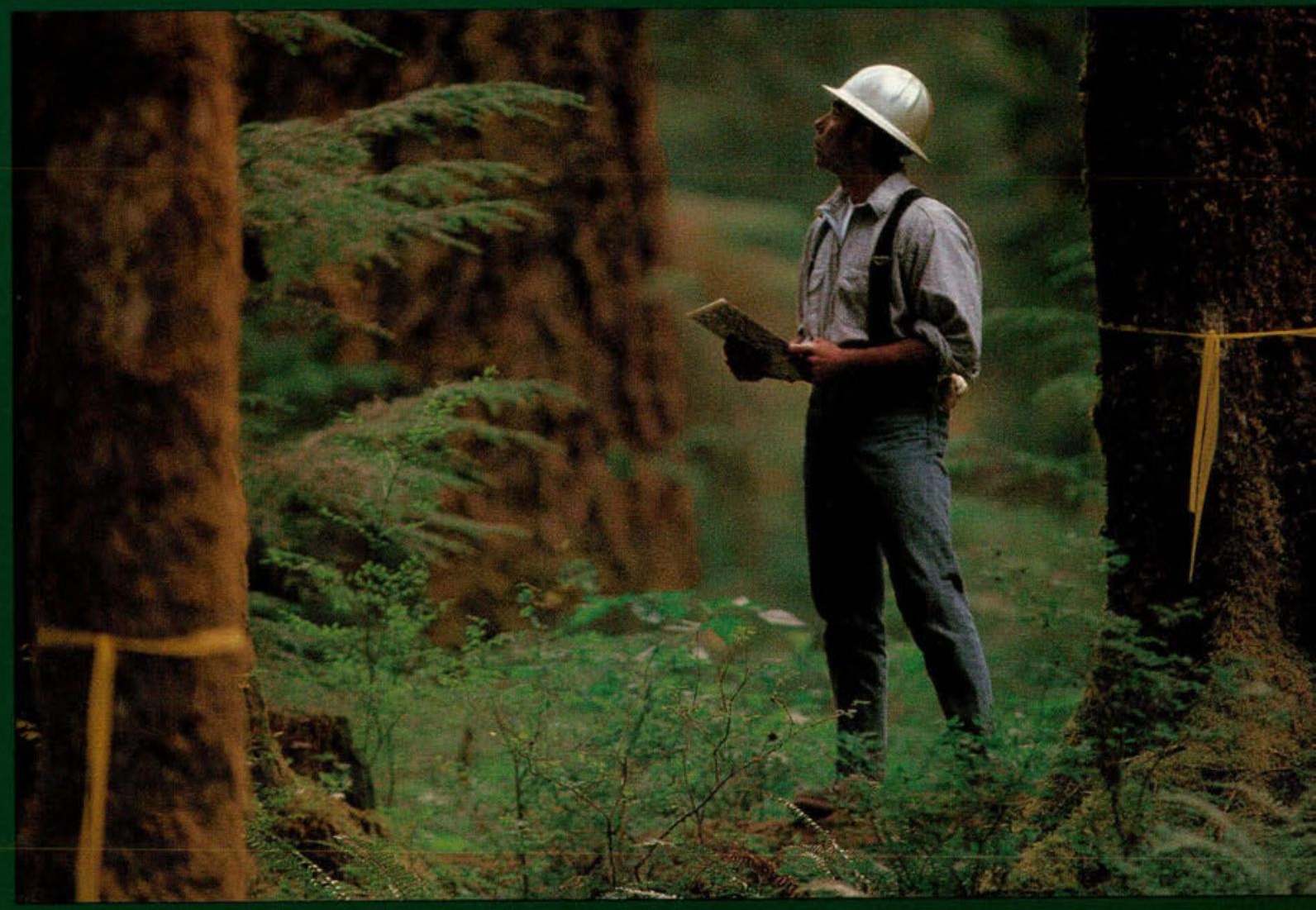

\section{THERAPY SHOULD NOT CHANGE THE WAY HE FEELS}

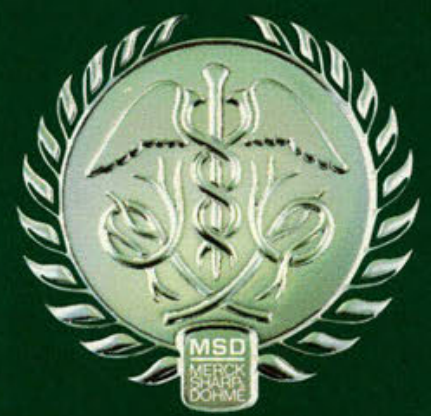




\section{LOWER HIS BLOOD PRESSURE, NOT HIS PERFORMANCE}

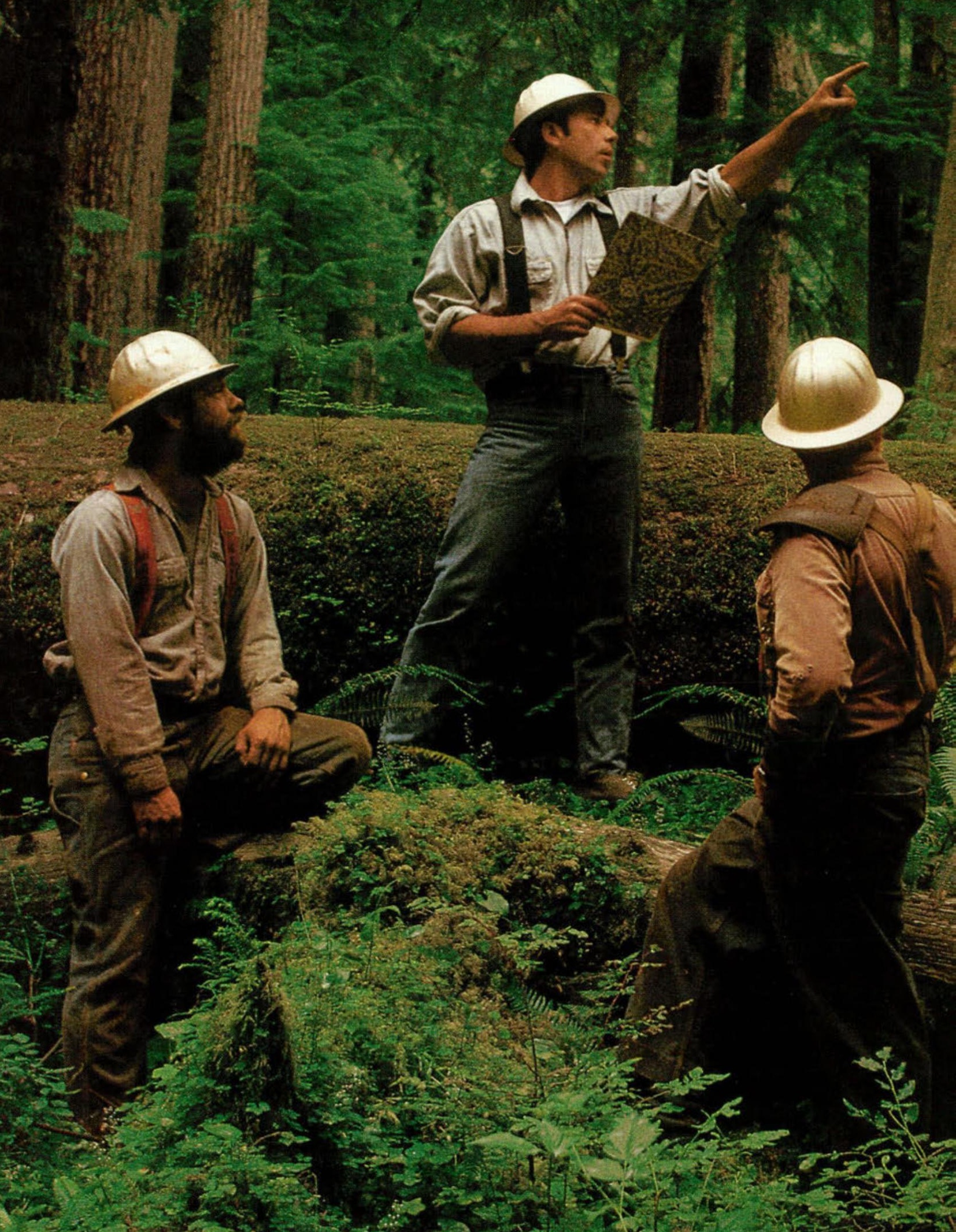




\section{FOR MANY HYPERTENSIVE PATIENTS START WITH ONCE-A-DAY}

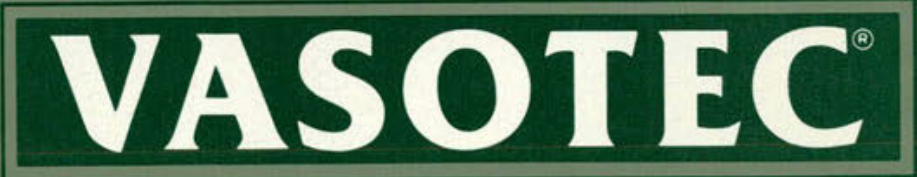

(ENALAPRIL MALEATE $\mid$ MSD)

\section{VASOTEC is generally well tolerated and not characterized}

by certain undesirable effects associated with selected

(4) agents in other antihypertensive classes. Thus, VASOTEC is being prescribed as initial therapy for hypertension

by an increasing number of physicians.

VASOTEC generally has little or no effect on a patient's physical or mental activity, since it is not characterized by certain subjective symptoms (such as malaise or drowsiness) which may interfere with such activity. This highly favorable tolerability profile may be related to the specificity of action of VASOTEC on the renin-angiotensinaldosterone system.

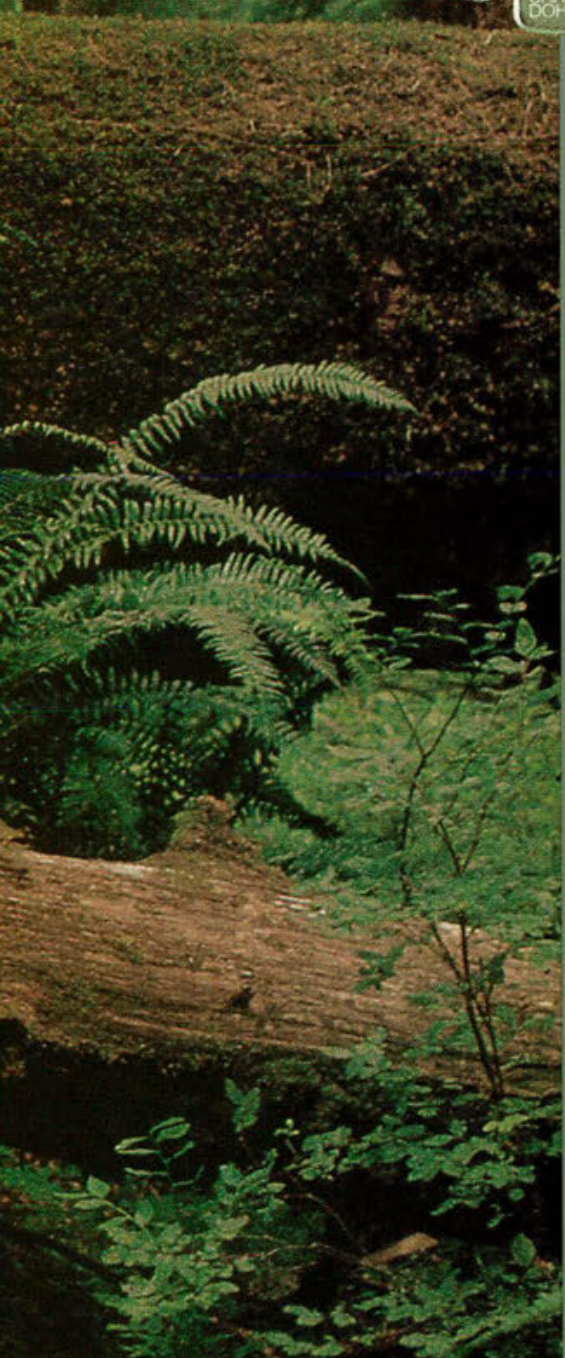

VASOTEC is contraindicated in patients who are hypersensitive to this product and in patients with a history of angioedema related to previous treatment with an ACE inhibitor.

Patients at risk for excessive hypotension, sometimes associated with oliguria and/or progressive azotemia and rarely with acute renal failure and/or death, include those with heart failure, hyponatremia, high-dose diuretic therapy. recent intensive diuresis or increase in diuretic dose, renal dialysis, or severe volume and/or salt depletion of any etiology. It may be advisable to eliminate the diuretic (except in heart failure patients), reduce the diuretic dose, or increase salt intake cautiously before initiating therapy with VASOTEC in patients at risk for excessive hypotension who are able to tolerate such adjustments. in patients at risk for excessive hypotension, therapy should be started under very close medical supervision, and such patients should be followed closely for the first two weeks of treatment and whenever the dose of enalapril and/or diuretic is increased.

In using VASOTEC, consideration should be given to the fact that another ACE inhibitor, captopril, has caused agranulocytosis, particularly in patients with renal impairment or collagen vascular disease, and the available data are insufficient to show that VASOTEC does not have a similar risk.

If angioedema of the face, extremities, lips, tongue, glottis, and/or larynx develops, VASOTEC should be promptly discontinued and the patient carefully observed until the swelling disappears. Angioedema associated with laryngeal edema may be fatal.

For more details, including information on treatment, see the Brief Summary of Prescribing Information on the last page of this advertisement. 


\section{VASOTEC (ENALAPRIL MALEATE $\mid$ MSD)}

VASOTEC is available in $2.5-\mathrm{mg}, 5-\mathrm{mg}, 10-\mathrm{mg}$, and $20-\mathrm{mg}$ tablet strengths

Contraindications: VASOTEC ${ }^{\circ}$ (Enalapril Maleate, MSD) is contraindicated in patients who are hypersensitive to this product and in patients with a history of angioedema related to previous treatment with an ACE inhibito:

Warnings: Angioedema: Angioedema of the lace, extremities, lips, tongue, glottis, and/or larynx has been reported in patientstreated with ACE inhibitors, including VASOTEC. In suchcases. VASOTEC should bepromptly discontinuedand the patient carefully observed until the swelling disappears. In instances where swelling has been confined to the face and lips. the condition has generally resolved without treatment, although antihistamines have been useful in relieving symptoms. Angioedema associated with laryngeal edema may be fatal. Where there is involvement of the tongue, glottis, or larynx likely to cause airway obstruction, appropriate therapy, e.g., subcutaneous epinephrine solution $1: 1000(0.3 \mathrm{~mL}$ to $0.5 \mathrm{~mL})$, should be promptly administered. (See ADVERSE REACTIONS.)

Hypotension: Excessive hypotension is rare in uncomplicated hypertensive patients treated with VASOTEC alone. Hea tailure patients given VASOTEC commonly have some reduction in blood pressure, especially with the first dose, bu discontinuation of therapy for continuing symptomatic hypotension usually is not necessary when dosing instruction are followed, caution should be observed when initiating therapy. (See DOSAGE AND ADMINISTRATION.) Patients at risk for excessive hypotension, sometimes associated with oliguria and/or progressive azotemia and rarely with acute renal failure and/or death, include those with the following conditions or characteristics: heart failure, hyponatremia high-dose diuretic therapy. recent intensive diuresis or increase in diuretic dose, renal dialysis, or severe volume and/or salt depletion of any etiology. It may be advisable to eliminate the diuretic (except in heart tailure patients), reduce the diuretic dose, or increase salt intake cautiously before initiating therapy with VASOTEC in patients at risk for excessive hypotension who are able to tolerate such adjustments. (See PRECAUTIONS, Drug Interactions and ADVERSE REAC TIONS.) In patients at risk for excessive hypotension, therapy should be started under very close medical supervision and such patients should be followed closely for the first two weeks of treatment and whenever the dose of enalapri and/or diuretic is increased. Similar considerations may apply to patients with ischemic heart disease or cardiovascular disease in whom an excessive fall in blood pressure could result in a myocardial infarction or cerebrovascular accident. If excessive hypotension occurs, the patient should be placed in supine position and, if necessary, receive an intravenous infusion of normal saline. A transient hypotensive response is not a contraindication to further doses of VASOTEC, which usually can be given without difficulty once the blood pressure has stabilized. If symptomatic hypotension develops, a dose reduction or discontinuation of VASOTEC or concomitant diuretic may be necessary

Neutropenia/Agranulocytosis: Another ACE inhibitot, captopril, has been shown to cause agranulocytosis and bone marrow depression, rarely in uncomplicated patients but more frequently in patients with renal impairment, especially if they also have a collagen vascular disease. Available data from clinical trials of enalapril are insufficient to show that enalapri does not cause agranulocytosis at similar rates. Foreign marketing experience has revealed several cases of neutropenia or agranulocytosis in which a causal relationship to enalapril cannot be excluded. Periodic monitoring of white blood cell counts in patients with collagen vascular disease and renal disease should be considered

Precautions: General: Impaired Renal Function: As a consequence of inhibiting the renin-angiotensin-aldosterone system, changes in renal function may be anticipated in susceptible individuals. In patients with severe heart tailure whose renal function may depend on the activity of the renin-angiotensin-aldosterone system, treatment with ACE inhibitors, including VASOTEC, may be associated with oliguria and/or progressive azotemia and rarely with acute renal tailure and/or death

In clinical studies in hypertensive patients with unilateral or bilateral renal artery stenosis, increases in blood urea nitrogen and serum creatinine were observed in $20 \%$ of patients. These increases were almost always reversible upon discontinuation of enalapril and/or diuretic therapy In such patients, renal function should be monitored during the first lew weeks of therapy

Some patients with hypertension or heart tailure with no apparent preexisting renal vascular disease have developed increases in blood urea and serum creatinine, usually minor and transient, especially when VASOTEC has been given concomitantly with a diuretic. This is more likely to occur in patients with preexisting renal impairment. Dosage reduction and/or discontinuation of the diuretic and/Or VASOTEC may be required.

Evaluation of patients with hypertension or heart failure should always include assessment of renal function. (SEe DOSAGE AND ADMINISTRATION )

Hyperkalemia: Elevated serum potassium ( $>5.7 \mathrm{mE} / \mathrm{L}$ ) was observed in approximately $1 \%$ of hyperlensive patients in clinical trials. In most cases these were isolated values which resolved despite continued therapy Hyperkalemia was cause of discontinuation of therapy in $0.28 \%$ of hypertensive patients. In clinical trials in heart failure, hyperkalemia was observed in $3.8 \%$ of patients, but was not a cause for discontinuation

Risk factors for the development of hyperkalemia include renal insulficiency, diabetes mellitus, and the concomitant use of potassium-sparing diuretics, potassium supplements, and/or potassium-containing salt substitutes, which should be used cautiously, if at all, with VASOTEC. (See Drug Interactions.) Surgery/Anesthesia: In patients undergoing major surgery or during anesthesia with agents that produce hypotension. considered to be due to this mechanism, it can be corrected by volume expansion.

Information for Patients:

Angioedema: Angioedema, including laryngeal edema, may occur especially following the first dose of enalapril. Patients should be so advised and told to report immediately any signs or symptorns suggesting angioederna (swelling of face, extremities, eyes, lips, tongue, difficulty in swallowing or breathing) and to take no more drug until they have consulted with the prescribing physician.

Hypotension: Patients should be cautioned to report lightheadedness especially during the first tew days of therapy. If actual syncope occurs, the patients should be told to discontinue the drug until they have consulted with the prescribing physician.

All patients should be cautioned that excessive perspiration and dehydration may lead to an excessive fall in blood pressure because of reduction in fluid volume. Other causes of volume depletion such as vomiting or diarrhea may also lead to a tall in blood pressure; patients should be advised to consult with the physician

Hyperkalemia: Patients should be told not to use salt substitutes containing potassium without consulting their physician.

Neutropenia: Patients should be told to report promptly any indication of infection (e.g. sore throat, fever) which may be a sign of neutropenia.

NOTE: As with many other drugs, certain advice to patients being treated with enalapril is warranted. This intormation is intended to aid in the sate and effective use of this medication. It is not a disclosure of all possible adverse or intended effects.

Drug Interactions:

Hypotension: Patients on Diuretic Therapy: Patients on diuretics and especially those in whom diuretic therapy was recently instituted may occasionally experience an excessive reduction of blood pressure after initiation of therapy with enalapril. The possibility of hypotensive effects with enalapril can be minimized by either discontinuing the diuretic or increasing the salt intake prior to initiation of treatment with enalaprit. If it is necessary to continue the diuretic, provide close medical supervision after the initial dose for at least two hours and until blood pressure has stabilized for at least an additional hout (See WARNINGS and DOSAGE AND ADMINISTRATION.)

Agents Causing Renin Release: The antihypertensive effect of VASOTEC is augmented by antihypertensive agents that cause renin release (e.g. diuretics)

Other Cardiovascular Agents: VASOTEC has been used concomitantly with beta-adrenergic-blocking agents, methyldopa, nitrates, calcium-blocking agents, hydralazine, prazosin, and digoxin without evidence of clinically significant adverse interactions.

Agents Increasing Serum Potassium: VASOTEC attenuates potassium loss caused by thiazide-type diuretics. Potassium-sparing diuretics (e.g. spironolactone, triamterene, or amiloride), potassium supplements, or potassium-containing salt substitutes may lead to significant increases in serum potassium. Therefore, if concomitant use of these agents is indicated because of demonstrated hypokalemia. they should be used with caution and with frequent monitoring of serum potassium. Potassium-sparing agents should generally not be used in patients with heart failure receiving VASOTEC

Lithium: A few cases of lithium toxicity have been reported in patients receiving concomitant VASOTEC and lithium and were reversible upon discontinuation of both drugs. Although a causal relationship has not been established, it is recommended that caution be exercised when lithium is used concomitantly with VASOTEC and serum lithium levels should be monitored trequently
Pregnancy - Category C: There was no fetotoxicity or teratogenicity in rats treated with up to $200 \mathrm{mg} / \mathrm{kg} /$ day of en (333 times the maximum human dose). Fetotoxicity, expressed as a decrease in average fetal weight, occurred given $1200 \mathrm{mg} / \mathrm{kg} /$ day of enalapril but did not occur when these animals were supplemented with saline. Enalap not teratogenic in rabbits. However, maternal and fetal toxicity occurred in some rabbits at doses of $1 \mathrm{mg} / \mathrm{kg}$ more. Saline supplementation prevented the maternal and tetal toxicity seen at doses of 3 and $10 \mathrm{mg} / \mathrm{kg} / \mathrm{day}$, but $30 \mathrm{mg} / \mathrm{kg} /$ day ( 50 times the maximum human dose)

Radioactivity was found to cross the placenta following administration of labeled enalapril to pregnant hamsters There are no adequate and well-controlled studies in pregnant women. VASOTEC $*$ (Enalapril Maleate, MSD) st used during pregnancy only if the potential benefit justifies the potential risk to the tetus.

Nursing Mothers: Milk in lactating rats contains radioactivity following administration of $14 \mathrm{C}$ enalapril maleate. known whether this drug is secreted in human milk. Because many drugs are secreted in human milk. caution shou exercised when VASOTEC is given to a nursing mother.

Pediatric Use: Safety and effectiveness in children have not been established.

Adverse Reactions: VASOTEC has been evaluated for satety in more than 10,000 patients, including ov patients treated for one year or more. VASOTEC has been found to be generally well tolerated in controlled clinical involving 2987 patients.

$(43 \%)$, and tatigue $(3 \%)$.

Other adverse experiences occurring in greater than $1 \%$ of patients treated with VASOTEC in controlled clinical were: diarthea $(1.4 \%)$, nausea $(1.4 \%)$, rash $(1.4 \%)$, cough $(1.3 \%)$, orthostatic effects $(1.2 \%)$, and asthenia $(1.1 \%)$. Heart Failure: The most frequent clinical adverse experiences in both controlled and uncontrolled trials were: dizz
$(79 \%)$, hypotension $(6.7 \%)$, orthostatic effects $(2.2 \%)$, syncope $(22 \%)$, cough $(2.2 \%)$, chest pain $(2.1 \%)$, and dia

Other adverse experiences occurring in greater than $1 \%$ of patients treated with VASOTEC in both controlled and ur trilled clinical trials were: fatigue $(1.8 \%)$, headache $(1.8 \%)$, abdominal pain $(1.6 \%)$, asthenia $(1.6 \%)$, orthostatic tension $(1.6 \%)$, vertigo $(1.6 \%)$, angina pectoris $(1.5 \%)$, nausea $(1.3 \%)$, vomiting $(1.3 \%)$, bronchitis $(1.3 \%)$, dys $(1.3 \%)$, urinary tract infection (1.3\%), rash (1.3\%), and myocardial infarction (1.2\%).

Other serious clinical adverse experiences occurring since the drug was marketed or adverse experiences occurr $0.5 \%$ to $1 \%$ of patients with hypertension or heart tailure in clinical trials in order of decreasing severity within category:

Cardiovascular: Myocardial infarction or cerebrovascular accident, possibly secondary to excessive hypotensi. high-risk patients (see WARNINGS. Hypotension); cardiac arrest; pulmonary embolism and intarction; rhythm bances: atrial fibrillation; palpitation.

Digestive: Ileus, pancreatitis, hepatitis or cholestatic jaundice, melena, anorexia, dyspepsia, constipation, glossit Nervous/Psychiatric: Depression, confusion, ataxia, somnolence, insomnia, nervousness, paresthesia. Urogenital: Renal tailure, oliguria, renal dystunction (see PRECAUTIONS and DOSAGE AND ADMINISTRATION), tate hypertrophy

Respiratory: Bronchospasm, rhinorrthea, asthma, upper respiratory infection.

Skin: Herpes zostet, pruritus, alopecia, flushing, photosensitivity

Other: Muscle cramps, hyperhidrosis, impotence, blurred vision, taste alteration, tinnitus

A symptom complex has been reported which may include fevet, myalgia, and arthralgia; an elevated erythrocyte mentation rate may be present. Rash or other dermatologic manilestations may occur. These symptoms have peared after discontinuation of therapy

Angioedema: Angioedema has been reported in patients receiving VASOTEC $(0.2 \%)$. Angioederma associated laryngeal edema may be fatal. If angioedema of the face, extremities, lips, tongue, glottis, and/or larynx occurs, ment with VASOTEC should be discontinued and appropriate therapy instituted immediately. (See WARNINGS. Hypotension: In the hypertensive patients, hypotension occurred in $0.9 \%$ and syncope occurred in $0.5 \%$ of pat following the initial dose or during extended therapy Hypotension or syncope was a cause for discontinuation of the
in $0.1 \%$ of hypertensive patients. In heart tailure patients, hypotension occurred in $6.7 \%$ and syncope occurred in in $0.1 \%$ of hypertensive patients. In heart tailure patients, hypotension occurred in $6.7 \%$ and syncope occurred in 2
of patients. Hypotension or syncope was a cause for discontinuation of therapy in $1.9 \%$ of patients with heart ta (See WARNINGS.)

Clinical Laboratory Test Findings

Serum Electrolytes: Hyperkalemia (see PRECAUTIONS), hyponatremia

Creatinine, Blood Urea Nitrogen: In controlled clinical trials, minor increases in blood urea nitrogen and serum ci nine, reversible upon discontinuation of therapy were observed in about $0.2 \%$ of patients with essential hyperter treated with VASOTEC alone Increases are more likely to occur in patients receiving concomitant diuretics or in pat with renal artery stenosis. (See PRECAUTIONS.) In patients with heart failure who were also receiving diuretics w without digitalis, increases in blood urea nitrogen or serum creatinine, usually reversible upon discontinuatic VASOTEC and/or other concomitant diuretic therapy, were observed in about $11 \%$ of patients. Increases in blood nitrogen or creatinine were a cause for discontinuation in $1.2 \%$ of patients

Hemoglobin and Hematocrit: Small decreases in hemoglobin and hematocrit (mean decreases of approximately 0.3 and $1.0 \mathrm{vol} \%$, respectively) occur trequently in either hypertension or heart failure patients treated with VASOTEC b. rately of clinical importance unless another cause of anemia coexists. In clinical trials, less than $0.1 \%$ of patients dis tinued therapy due to anemia

Other (Causal Relationship Unknown):
marrow depression have been reported

.

Dosage and Administration: Hypertension: In patients who are currently being treated with a diuretic, symptor hypotension occasionally may occur following the initial dose of VASOTEC. The diuretic should, if possible be dis tinued for two to three days belore beginning therapy with VASOTEC to reduce the likelithood of hypotension. WARNINGS.) If the patient's blood pressure is not controlled with VASOTEC alone, diuretic therapy may be resum If the diuretic cannot be discontinued, an initial dose of $2.5 \mathrm{mg}$ should be used under medical supervision for at leas hours and until blood pressure has stabilized tor at least an additional hout (See WARNINGS and PRECAUTIONS, Interactions.)

The recommended inifial dose in patients not on diuretics is $5 \mathrm{mg}$ once a day Dosage should be adjusted accord blood pressure response. The usual dosage range is $101040 \mathrm{mg}$ per day administered in a single dose or in two div doses. In some patients treated once daily, the antihypertensive effect may diminish toward the end of the dosing int n such patients, an increase in dosage or twice-daily administration should be considered if blood pressure is not trolled with VASOTEC alone, a diuretic may be added.

Concomitant administration of VASOTEC with potassium suppiements, potassium salt substitutes, or potassiuming diuretics may lead to increases of serum potassium (see PRECAUTIONS).

Dosage Adjustment in Hypertensive Patients with Renal Impairment: The usual dose of enalapril is recommende natients with a creatinine clearance $>30 \mathrm{~mL} / \mathrm{min}$ (serum creatinine of up 10 approximately $3 \mathrm{mg} / \mathrm{dL}$ ). For patients creatinine clearance $\leqslant 30 \mathrm{~mL} / \mathrm{min}$ (serum creatinine $\geqslant 3 \mathrm{mg} / \mathrm{dL}$ ), the first dose is $2.5 \mathrm{mg}$ once daily The dosage $\mathrm{me}$ titrated upward until blood pressure is controlled or to a maximum of $40 \mathrm{mg}$ daily

Heart Failure: VASOTEC is indicated as adjunctive therapy with diuretics and digitalis. The recommended starting do $2.5 \mathrm{mg}$ once or twice daily After the initial dose of VASOTEC, the patient should be observed under medical superv or at least two hours and until blood pressure has stabilized for at least an additional hour. (See WARNINGS and CAUTIONS, Drug Interactions.) If possible, the dose of the diuretic should be reduced, which may diminish the likelit of hypotension. The appearance of hypotension after the initial dose of VASOTEC does not preclude subsequent ca tive management of the hypotension. The usual therapeutic dosing rang ily given in two divided doses. The maximum daily dose is $40 \mathrm{mg}$. Oncea controlled study, but nearly all patients in this study were given $40 \mathrm{mg}$, the maximum e has been much more experience with twice-daily dosing. In addition, in a placeborolled study which demonstrated reduced mortality in patients with severe heart failure (NYHA Class IV), patients treated with 2.5 to $40 \mathrm{mg}$ per day of VASOTEC, almost always administered in two divided doses. (See CLINICAL Ph MACOLOGY, Pharmacodynamics and

Dosage Adjustment in Heart Failure Patients with Renal Impairment or Hyponatremia: In heart failure patients hyponatremia (serum sodium $<130 \mathrm{mEq} / \mathrm{L}$ ) or with serum creatinine $>1.6 \mathrm{mg} / \mathrm{dL}$, therapy should be initiated at 2 s daily under close medical supervision. (See DOSAGE AND ADMINISTRATION. Heart Failure. WARNINGS, and CAUTIONS. Drug interactions.) The dose may be increased to $2.5 \mathrm{mg}$ b.i.d., then $5 \mathrm{mg}$ b.i.d. and higher as needed, usually at intervals of tour days or more, if at the time of dosage adjustment there is not excessive hypotension or significant deterioration of renal function. The maximum daily dose is $40 \mathrm{mg}$ for more detailed information, consult your MSD representative or see Prescribing Information. Merck SHAR Sharp \& Dohme, Division of Merck \& Co., INC., West Point, PA $19486 . \quad$ J8vS36(815) DOHM 